\title{
VISUAL ORBIT OF THE LOW-MASS BINARY GJ 164 AB
}

\author{
Frantz Martinache $^{1,2}$, Barbara Rojas-Ayala ${ }^{1}$, Michael J. Ireland ${ }^{3}$ James P. Lloyd $^{1}$, And Peter G. Tuthill ${ }^{3}$ \\ ${ }^{1}$ Department of Astronomy, Cornell University, Ithaca, NY, USA \\ ${ }^{2}$ Subaru Telescope, Hilo, HI, USA \\ ${ }^{3}$ School of Physics, University of Sydney, Sydney, NSW, Australia \\ Received 2008 September 4; accepted 2009 January 30; published 2009 April 6
}

\begin{abstract}
We report seven successful observations of the astrometric binary GJ 164 AB system with aperture masking interferometry. The companion, with a near-infrared contrast of 5:1 was detected beyond the formal diffraction limit. Combined with astrometric observations from the literature, these observations fix the parallax of the system, and allow a model-independent mass determination of both components. We find the mass of GJ 164 B to be $0.096 \pm 0.008 M_{\odot}$. With data taken with the newly commissioned TripleSpec spectrograph, we examine GJ 164's near-infrared spectral features and compare them to a sample of M dwarfs of known metallicity. Models are not consistent with color and mass, requiring a very young age to accommodate a secondary too luminous, a scenario ruled out by the kinematics.
\end{abstract}

Key words: stars: low-mass, brown dwarfs - techniques: high angular resolution - techniques: spectroscopic

\section{INTRODUCTION}

The development of a self-consistent theory of the internal structure of stars and the construction of models which trace evolutionary behavior are major achievements of modern astrophysics. Both theory and models have however long been centered on intermediate- and high-mass stars, with little consideration of objects with masses below $0.6 M_{\odot}$. The realization that the solar neighborhood is overwhelmingly dominated by low-mass stars (van de Kamp 1971; Henry 1998), and the discovery of brown dwarfs (Nakajima et al. 1995) have brought a lot more attention to the lower end of the main sequence and what lies below.

The complex physical nature of these very low mass objects makes their modeling intricate. Boundaries between stars, brown dwarfs, and planets, as well as theoretical relations that predict the luminosity and temperature as functions of age, mass, and metallicity, are still largely untested in the relevant range of parameters. Correct calibration of these models is of extreme importance, since the same self-consistent theory is used to estimate the mass of brown dwarfs (Burrows et al. 2006) and giant exoplanets (Baraffe et al. 2003) from their luminosity and age.

The observations required to challenge and improve the models are dynamical mass measurements of multiple star systems, combined with accurate photometry and distance determination. Besides gravitational microlensing (Paczynski 1986), the observation of binary systems and the use of Newtonian orbital dynamics provide the only method of directly measuring accurate stellar masses. With maximum sensitivity at separations greater than 1 arcsec, conventional Adaptive Optics (AO) observations at Palomar are limited to the most nearby objects or the consequent orbital periods become too long to lead to dynamical masses. We have therefore been using aperture masking interferometry (Tuthill et al. 2000) in conjunction with AO (Lloyd et al. 2006). The precision calibration of the data achieved with this observing mode indeed leads to reliable results up to and beyond the formal diffraction limit (super resolution), and very precise photometry. Combined with radial velocity (Martinache et al. 2007) or astrometry (Ireland et al. 2008), aperture masking interferometry has so far provided some of the most precise (dynamical) masses of objects below $0.1 M_{\odot}$.

\section{CHARACTERIZATION OF THE ORBIT}

We report here aperture masking interferometry observations of the astrometric binary GJ 164 AB. GJ 164 (aka LHS 1642, G 175-19 or Ross 28) is a high proper-motion star, cataloged as an M4.5 dwarf (Reid et al. 1995), 11-13 pc distant. From the Two Micron All Sky Survey (2MASS) catalog, its apparent near-infrared magnitudes are $J=8.773 \pm 0.032$, $H=8.248 \pm 0.030$, and $K=7.915 \pm 0.016$. The discovery of GJ 164 B was reported by Pravdo et al. (2004), as a part of the STEPS program of Pravdo \& Shaklan (1996). The combination of the astrometry data from the discovery paper, combined with the aperture masking interferometry data reported in this paper provides a complete dynamical characterization of the system, resulting in masses that are independent of the use of a model or a mass-luminosity relation (MLR). GJ 164 is an especially interesting target for the mass of the B component lies very close to the substellar limit.

\subsection{Aperture Masking Interferometry}

Measuring the dynamical masses of a low-mass binary system requires some patience, for the observations have to cover at the very least a significant fraction of the orbital period. Because of its low mass (less than $0.6 M_{\odot}$ ), a relatively short period (less than $10 \mathrm{yr}$ ) binary will have a semimajor axis smaller than 4 AU. Even for objects as close as $10 \mathrm{pc}$, the angular separation will not exceed 0.4 arcsec, a performance theoretically within the grasp of a 5-10 m class telescope equipped with AO. Yet, in practice, the residual quasi-static speckles inthe AO point-spread function (PSF) halo seriously limit the capability of detecting a companion at angular separation smaller than $2-4 \lambda / D$ (Racine et al. 1999). Aperture masking interferometry with AO addresses this issue.

A nonredundant mask discards most of the pupil by sampling a few spatial frequencies only (Readhead et al. 1988; Nakajima et al. 1989). One admittedly loses most of the light (the 9-hole mask used for this works transmits approximately 15\%) but rejects all atmospheric noise, as well as internal aberrations and noncommon path errors, which in turn, dramatically increases the signal-to-noise ratio (Tuthill et al. 2000). Used in conjunction, aperture masking interferometry and AO provide stable fringes, and enable long integration times, therefore making 
Table 1

Aperture Masking Measurements at Palomar and Keck: Angular Separation and Position Angle of GJ 164 B

\begin{tabular}{lclcr}
\hline \hline $\begin{array}{l}\text { Julian Date } \\
(-2,450,000)\end{array}$ & Band & Telescope & $\begin{array}{c}\text { Sep. } \\
\text { (mas) }\end{array}$ & $\begin{array}{c}\text { PA } \\
\text { (degrees) }\end{array}$ \\
\hline 3004.85 & $H$ & Palomar & $82.8 \pm 1.6$ & $111.1 \pm 0.9$ \\
3632.96 & $H$ & Palomar & $53.3 \pm 1.3$ & $149.1 \pm 1.2$ \\
3723.66 & $H$ & Palomar & $80.1 \pm 3.3$ & $116.1 \pm 2.1$ \\
3779.71 & $H, K_{S}$ & Palomar & $86.1 \pm 2.0$ & $102.1 \pm 0.9$ \\
3957.62 & $H$ & Keck & $49.3 \pm 0.5$ & $47.0 \pm 0.6$ \\
4018.98 & $K_{S}$ & Palomar & $39.4 \pm 1.3$ & $344.8 \pm 2.6$ \\
4078.79 & $K_{S}$ & Palomar & $57.3 \pm 2.2$ & $294.1 \pm 2.8$ \\
\hline
\end{tabular}

Table 2

GJ 164 Photometry

\begin{tabular}{lcc}
\hline \hline $\begin{array}{l}\text { Julian Date } \\
(-2,450,000)\end{array}$ & Filter & Contrast \\
\hline 3004.85 & & $5.97 \pm 0.32$ \\
3632.96 & $H$ & $5.77 \pm 0.46$ \\
3723.67 & $H$ & $5.78 \pm 0.63$ \\
3779.71 & $H$ & $5.53 \pm 0.60$ \\
& $K_{S}$ & $4.89 \pm 0.19$ \\
3957.62 & $H$ & $5.42 \pm 0.03$ \\
4018.98 & $K_{S}$ & $8.66 \pm 4.81$ \\
& $H$ & $6.38 \pm 1.65$ \\
4078.79 & $K_{S}$ & $5.67 \pm 1.17$ \\
\hline
\end{tabular}

faint target accessible. The only limit is whether the AO system can lock on the system. Thus, any target that can be observed with AO can also be observed with aperture masking interferometry. With a visible magnitude $V=13.5$ (Weis 1996), GJ 164 can fairly easily be observed with aperture masking at Palomar and Keck.

The PSF of a nonobscurated telescope of diameter $D$ is described by the radial Airy function, and a rule of thumb known as the Rayleigh criterion states that two sources are resolved if separated by at least $1.22 \lambda / D$ (the first zero of the Airy function). One obvious advantage of aperture masking interferometry over standard direct imaging is that in masking the entire pupil except for two diametrically opposed holes (therefore separated by the distance $D$ ), the system produces fringes of period $\lambda / D$. Two sources are now resolved if separated by more than $0.5 \lambda / D$, a condition also known as the Michelson criterion: masking the telescope offers a gain of 2.44 in resolution in a very straightforward manner.

Although useful for comparison, these criteria are however somewhat arbitrary and in practice, good calibration of the data in long baseline interferometry permits the resolution of structures smaller than $0.5 \lambda / D$. See, for instance, Figure 1 of Kervella et al. (2008) that shows structures smaller than 2 mas being resolved by a $\sim 30 \mathrm{~m}$ baseline $B$ for $\lambda=2 \mu \mathrm{m}$, i.e., $\lesssim 0.15 \lambda / B$.

The data presented in Tables 1 and 2 show that the companion to GJ $164 \mathrm{~A}$ is detected on multiple occasions beyond the formal diffraction limit, in what is sometimes called the super-resolution regime. Tuthill et al. (2006) provide a recent description of the general principles and performances of aperture masking interferometry, while Lloyd et al. (2006) detail the experiment undertaken at Palomar, and Kraus et al. (2008) the aperture masking mode of NIRC2.

The AO observations performed with PHARO (Hayward et al. 2001) at Palomar as well as with NIRC2 at Keck II span the

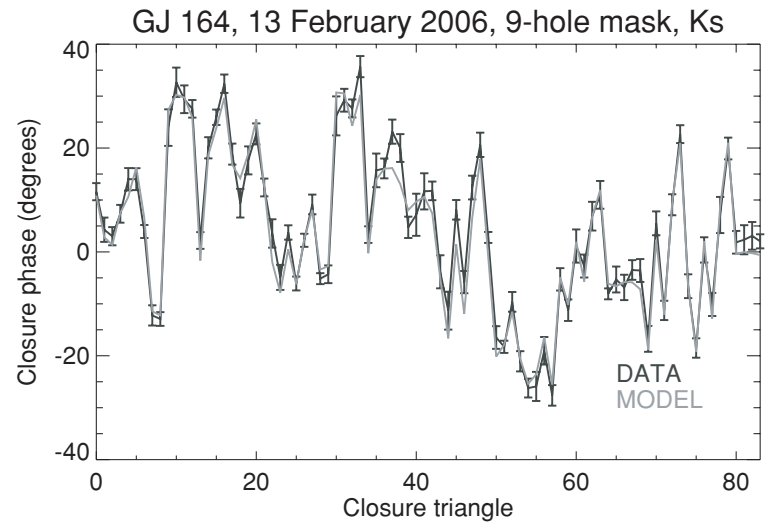

Figure 1. Calibrated closure phases observed on JD 2,453,779.71 with a $K_{S}$ filter. A binary star model (light gray) with an 86 mas separation, PA of $102^{\circ}$, and a contrast of 5:1 satisfactorily matches the observations (dark gray). For an unresolved system, each closure phase would be either $0^{\circ}$ or $180^{\circ}$ with the same confidence intervals.

range 2003 December to 2006 December. The companion was successfully detected all seven runs. The data are calibrated with observations of one or several stars of similar brightness for both the $\mathrm{AO}$ wave front sensor and the science camera to ensure comparable wave front correction and signal-to-noise ratio. The final data product of our custom software pipeline written in IDL, is a collectionof calibrated closure phases (Baldwin et al. 1986). This very robust observable rejects both atmospheric noise and calibration errors of the wave front sensor.

Each closure phase is a measure of the degree of asymmetry of the target for a given position angle (PA). Thus, a symmetric object like a point source or a near-equal binary exhibits $0^{\circ}$ or $180^{\circ}$ closure phase (Monnier 2000), while a nonequal binary exhibits closure phase of intermediate value. The 9hole mask provides the simultaneous measurement of $\left(\begin{array}{l}9 \\ 3\end{array}\right)=84$ possible closure triangles, $\left(\begin{array}{l}8 \\ 2\end{array}\right)=28$ of which are theoretically independent. Figure 1 represents one such set of closure phases observed with the 9 -hole mask in the $K$ band.

The instantaneous geometry of a binary star is modeled by a set of three parameters: contrast ratio $c$, angular separation $a$, and PA $\theta$. The example of one such model fitted to a set of closure phases is given in Figure 1. The aperture masking data processing software typically uses several hundreds of frames which are averaged to produce the closure phases. Due to the central limit theorem, the distribution of closure phases $\left\{\phi_{i}\right\}$ can be considered Gaussian, with uncertainties $\sigma_{i}$ given by the standard deviation to the mean. The best fit minimizes the traditionally used goodness-of-fit parameter $\chi^{2}$ defined as

$$
\chi^{2} \equiv \sum_{i} \frac{\left(\phi_{i}-f_{i}(a, \theta, c)\right)^{2}}{\sigma_{i}^{2}}
$$

where $f_{i}$ designates the model. A systematic error term is added to the closure phase dispersion $\sigma_{i}$ to achieve a reduced $\chi_{v}^{2}=1$. The likelihood of the parameters given the set of closure phases $\left\{\phi_{j}\right\}$ is related to the $\chi^{2}$ by

$$
L\left(a, \theta, c \mid\left\{\phi_{j}\right\}\right) \propto \exp \left(-\chi^{2} / 2\right) .
$$

Normalized, $L$ is the full joint probability density function for all three parameters. The confidence interval of one individual parameter is calculated by integrating out the two others. For example, the marginal probability density function for the 
angular separation is calculated by integrating out the PA and the contrast ratio:

$$
p(a)=\int d \theta d c L(a, \theta, c) .
$$

While the PA is naturally well orthogonal to the angular separation and the contrast ratio, the extraction of the other two parameters is a sensitive function of the angular separation. For large separation $(\sim \lambda / D$ and beyond), one sees (see Tables 1 and 2 ) that the contrast is better constrained than for the detections at smaller separations. Pravdo et al. (2006) show that for such detections at small angular separations, there is degeneracy between contrast and separation (see Figure 7 of the quoted paper). One can take advantage of the better-resolved observations for which the contrast is well constrained (JD 2,453,957.62 for the $H$ band and JD 2,453,779.71 for the $K_{S}$ band) and adopt these contrasts for the other observations, to get a better constraint on the angular separation. The results of this analysis: relative position and photometry are gathered in Tables 1 and 2. This and the more or less favorable seeing conditions explain the variable confidence interval sizes associated with each run. Overall, this analysis achieves a precision $\gtrsim 1$ mas at separations as low as $\sim 40$ mas in the $K_{S}$ band $(0.5 \lambda / D)$ on good nights at Palomar.

\subsection{Astrometry}

GJ 164 was discovered as a binary in an astrometric survey by Pravdo \& Shaklan (1996) and Pravdo et al. (2004). While the interferometry resolves the binary and provides its instant geometric configuration, the astrometry records the position of a star's photocenter, measured relative to several more distant stars. Repeated observations reveal the star's proper motion as well as its parallax. Once these two effects are subtracted from the astrometric signal, any residual wobble of the photocenter can be attributed to the presence of one or more companions, massive enough to cause an observable shift in position. The orbit of the companions can then be fully characterized.

Astrometric data were extracted from the figures of Pravdo et al. (2004) then added the motion due to the proper motion and the parallax given in the paper's tables, while the corresponding Julian dates were taken from the telescope observing schedule and therefore have a \pm 2 day uncertainty. These extracted values are gathered in Table 3 . We initially assume a 2.0 mas uncertainty for each individual measurement, as suggested by the residuals of the analysis performed by Pravdo et al. (2004).

The astrometric residual wobble however remains a function of the fractional light of the secondary, $\beta=L_{2} /\left(L_{1}+L_{2}\right)$, with $L_{1}$ and $L_{2}$ respectively denoting the luminosity of the primary and the secondary, as well as the fractional mass of the secondary $f=m_{2} /\left(m_{1}+m_{2}\right)$. In the limit at which the light of the secondary is negligible, the photocentric orbit is identical to the Keplerian orbit of the primary around the actual center of mass of the system. If the luminosity of the secondary is not so negligible, then the photocentric semimajor axis $\alpha$ is reduced proportionally to $\beta$. The ratio of the photocentric orbit $\alpha$, and the Keplerian orbit $a$ is (McCarthy et al. 1988)

$$
\alpha / a=f-\beta .
$$

A relatively small-mass, nonluminous secondary, and a relatively large-mass, luminous secondary will therefore have identical astrometric signatures. The data presented in Section 2.1 however rule out the latter possibility. Indeed, the aperture masking interferometry data gathered in Table 2 provide the $H$ and
Table 3

GJ 164 Astrometry

\begin{tabular}{lcr}
\hline \hline $\begin{array}{l}\text { Julian Date } \\
(-2,450,000)\end{array}$ & $\begin{array}{c}\Delta \text { R.A. } \\
(\text { mas })\end{array}$ & $\begin{array}{r}\Delta \text { Decl. } \\
(\mathrm{mas})\end{array}$ \\
\hline 0801.5 & 0.00 & 0.00 \\
0801.5 & 0.00 & -2.80 \\
1088.3 & -147.91 & -646.96 \\
1189.0 & -328.82 & -874.15 \\
1189.0 & -324.82 & -874.05 \\
1189.0 & -325.32 & -871.65 \\
1436.5 & -457.34 & -1409.82 \\
1494.0 & -563.37 & -1519.60 \\
2657.5 & -1639.04 & -4132.08 \\
2889.5 & -1745.18 & -4629.59 \\
2889.5 & -1745.18 & -4627.29 \\
2889.5 & -1745.88 & -4625.69 \\
3030.5 & -2014.31 & -4953.05 \\
3030.5 & -2014.61 & -4957.45 \\
3051.5 & -2044.87 & -5016.85 \\
\hline
\end{tabular}

$K_{S}$-band contrast ratios, which are turned into the following differences of magnitude:

$$
\begin{aligned}
\Delta H & =1.835 \pm 0.006 \\
\Delta K_{S} & =1.721 \pm 0.097 .
\end{aligned}
$$

These contrast ratios can be used to decompose the 2MASS combined magnitudes of the binary into apparent magnitudes for individual components: $H=8.432 \pm 0.030$ and $K=$ $8.117 \pm 0.017$ for GJ $164 \mathrm{~A}, H=10.267 \pm 0.030$ and $K=9.834 \pm 0.038$ for GJ 164 B.

The corresponding $(H-K)$ color indices: $(H-K)_{A}=$ $0.315 \pm 0.034$ and $(H-K)_{B}=0.433 \pm 0.048$ alone, provide a parallax-independent observable. While the $(H-K)$ color index of the primary is well compatible with an M4.5 spectral type (Henry et al. 1994; Koornneef 1983), the secondary is very unlikely to be any earlier than M8. In fact, according to Table 5 of Kirkpatrick et al. (2000), GJ 164 B qualifies as an M8.5 dwarf. Liebert \& Gizis (2006) provide RI photometry for a sample of late $\mathrm{M}$ and $\mathrm{L}$ dwarfs. Out of their sample, we extract five M8.5 dwarfs, and can therefore estimate $\left\langle R-K_{S}\right\rangle=6.41 \pm 0.26$ for this spectral type. Combined with the apparent $K_{S}$ magnitude determined here, this provides an estimate of $R=16.24 \pm 0.26$ for GJ $164 \mathrm{~B}$, while $R=12.4$ for GJ 164 A. This leads to an $R$-band contrast ratio $c=33.5$, and to a fractional light of the secondary $\beta=0.029 \pm 0.007$.

\section{CHARACTERISTICS OF GJ $164 \mathrm{AB}$}

\subsection{Orbital Parameters}

The aperture masking observations gathered in Table 1 provide the instant position of the companion to the primary. These observations alone suffice to constrain all seven orbital elements of the binary, independently of the parallax, with the semimajor axis expressed in angular units.

We use a model identical to the one described in Section 3.1.2 of Martinache et al. (2007) to fit a 7-parameter model to 14 observables. The observables are two coordinates for seven aperture masking data points. The seven orbital parameters are: the angular semimajor axis $\alpha$, the eccentricity $e$, the longitude of the ascending node $\omega_{0}$, the inclination $i$, the argument of the periastron $\Omega_{1}$, the period $P$, and the epoch at periastron passage $T_{P}$. Our solution exhibits a final reduced $\chi_{v}^{2}=0.61$ 
Table 4

Orbital Elements

\begin{tabular}{lcc}
\hline \hline Parameter & AO Only & AO + STEPS \\
\hline$\alpha$ (mas) & $80.4 \pm 1.3$ & $80.5 \pm 1.2$ \\
$e$ & $0.157 \pm 0.012$ & $0.161 \pm 0.012$ \\
$i(\mathrm{deg})$ & $121.9 \pm 0.9$ & $121.9 \pm 0.8$ \\
$\Omega_{1}(\mathrm{deg})$ & $272.4 \pm 1.3$ & $271.8 \pm 1.2$ \\
$\omega_{o}($ deg $)$ & $314.7 \pm 5.0$ & $311.7 \pm 3.7$ \\
$P$ (days) & $734.3 \pm 4.6$ & $736.9 \pm 1.7$ \\
$T_{P}$ (reduced JD) & $1868 \pm 19$ & $1856 \pm 8$ \\
\hline
\end{tabular}

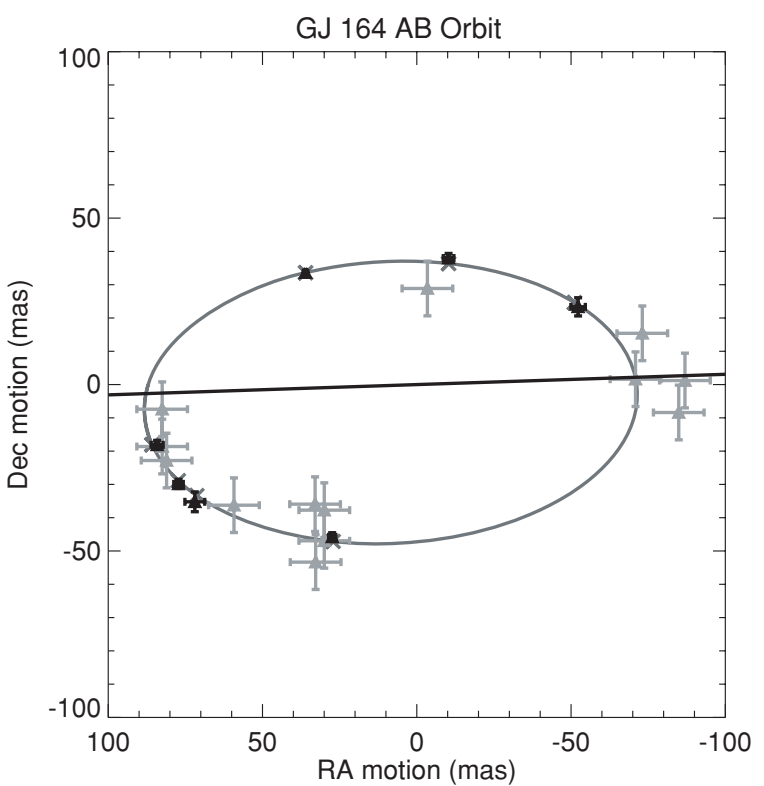

Figure 2. GJ 164 B ORBIT. The measurements and associated uncertainties of the aperture masking data are represented by dark gray rectangles. Light gray triangles represent the STEPS astrometry data. The relative apparent orbit (curve) is clockwise. Table 4 provides the corresponding orbital elements. The almost horizontal straight line represents the line of nodes.

for 7 degrees of freedom. Note that the temporal coverage of these observations definitely rules out the possibility of a $\sim 4 \mathrm{yr}$ orbital period, which although less likely, could not be excluded by Pravdo et al. (2004). The seven orbital parameters and the associated uncertainties are presented in Table 4.

We then proceed to a joined fit $\mathrm{AO}+$ astrometry, that is a 13parameter fit to 40 observables. The extra parameters included in this new analysis are: the two components of the proper motion, the relative parallax $\Pi$ as well as the semimajor axis of the motion of the primary around the center of mass of the system $a_{1}$. With a 2 mas uncertainty on the individual astrometric measurements, as assumed in Pravdo et al. (2004), the solution to this global fit exhibits a final reduced $\chi_{v}^{2}=1.04$ for 27 degrees of freedom. Table 4 compares the seven orbital parameters and their uncertainties obtained from the fit of the AO data only and the joined fit: both approaches produce compatible solutions. Figure 2 represents the orbit resulting from this joined fit, onto which are superposed the AO and astrometry data. Table 5 summarizes the astrometric elements re-derived from the joined fit.

The probability that a set of parameters is contained within a volume of the parameter space is proportional to the likelihood function, itself proportional to $\exp \left(-\chi^{2} / 2\right)$ (see Section 2.1). In an $n$-parameter fit, the marginal distribution for one parameter is calculated by integrating the likelihood over the $n-1$ other parameters. Tables 4 and 5 provide the $1 \sigma$ confidence
Table 5

Astrometric Elements

\begin{tabular}{lc}
\hline \hline Parameter & Value \\
\hline Proper motion R.A. (mas) & $-324.5 \pm 0.3$ \\
Proper motion decl. (mas) & $-808.3 \pm 0.3$ \\
Relative parallax (mas) & $70.0 \pm 0.8$ \\
Photocentric semimajor axis (mas) & $20.1 \pm 0.8$ \\
Total mass $\left(M_{\odot}\right)$ & $0.343 \pm 0.026$
\end{tabular}

Table 6

Dynamical Masses: Obtained from the Joined Analysis AO+Steps

\begin{tabular}{lc}
\hline \hline Parameter & Value \\
\hline Total mass & $M_{T}=0.343 \pm 0.026 M_{\odot}$ \\
Primary mass & $M_{1}=0.247 \pm 0.019 M_{\odot}$ \\
Secondary mass & $M_{2}=0.096 \pm 0.008 M_{\odot}$ \\
Mass ratio & $M_{2} / M_{T}=0.279 \pm 0.012$ \\
\hline
\end{tabular}

intervals for each parameter calculated this way. The method of Lampton et al. (1976) used by Pravdo et al. (2004) to calculate uncertainties however provides confidence intervals for the joined distribution, i.e., finds the hypercontour in the parameter space such that there is a $68.3 \%$ probability (i.e., $1 \sigma$ for a normal distribution) of having simultaneously all parameters within that hypercontour. The approach used in this work provides a significantly smaller confidence interval for each parameter (compare Table 5 with Table 1 of Pravdo et al. 2004). In the determination of uncertainties on the dynamical masses (see Section 3.2), this proves essential since the masses are only a function of subset of all the 13 parameters the joined fit gives.

\subsection{Dynamical Masses}

Like Pravdo et al. (2004), we use a $2 \pm 1$ mas correction to convert the relative parallax $\Pi=70.0 \pm 0.8$ mas, to the absolute parallax: $\Pi=72.0 \pm 1.2$ mas. We can now determine the actual relative semimajor axis of the binary: $a=1.1 \pm 0.2$ $\mathrm{AU}$, as well as the total mass:

$$
M_{T}=a^{3} / P^{2},
$$

that is, $M_{T}=0.343 \pm 0.026 M_{\odot}$. In the limiting case where the luminosity of the secondary is negligible, the fractional mass of the secondary (see Equation (4)) is given directly by the ratio of the photocentric and relative orbit semimajor axis, that is $\alpha / a=0.250 \pm 0.010$. Section 2.2 however shows the secondary is not negligible. The finite $R$-band contrast ratio translates into a fractional light $\beta=0.029 \pm 0.007$, which leads to the fractional mass $f=0.279 \pm 0.012$. Note that a remarkable feature of the fractional mass is that it is absolutely parallax-independent.

From the total mass and the fractional mass, one infers the masses of both components: $M_{1}=0.247 \pm 0.019$ and $M_{2}=0.096 \pm 0.008$. All dynamical characteristics of the GJ 164 system are gathered in Table 6.

To check a posteriori the validity of the uncertainties on the masses, we have conducted a series of Monte Carlo simulations. For each simulation, a new set of data (aperture masking and astrometry) was generated using a normal distribution centered on the original data points with standard deviation the associated uncertainties. The model-fit procedure highlighted in Section 3.1 was then used to determine the corresponding orbital and astrometric elements, which lead to the dynamical masses $M_{1}$ and $M_{2}$. Figure 3 presents the histograms of $M_{1}$ 

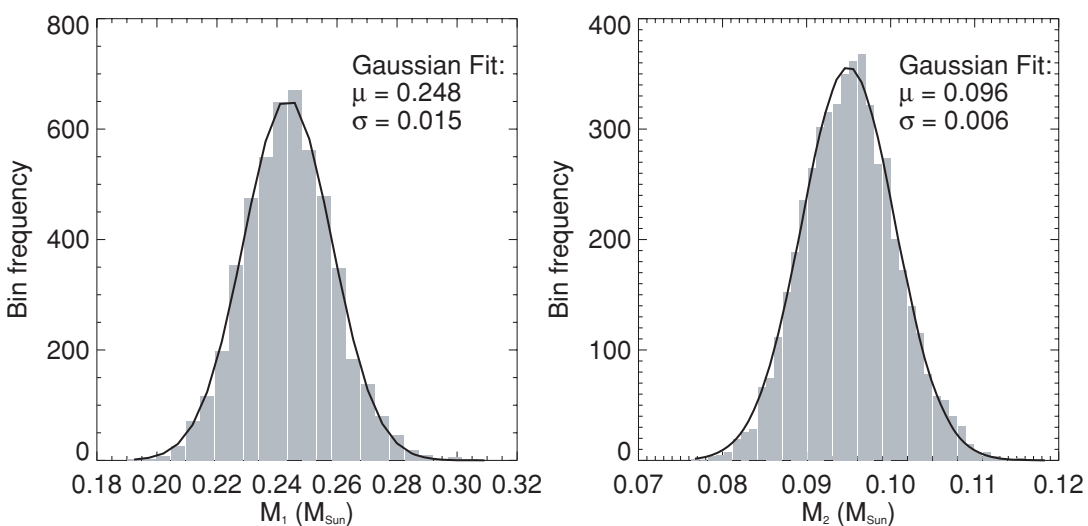

Figure 3. Histograms for $M_{1}$ and $M_{2}$ (gray bars) were calculated using a series of 5000 Monte Carlo experiments as described in Section 3.2. Each distribution is fitted with a Gaussian (solid black line) whose parameters (mean $\mu$ and standard deviation $\sigma$ ) are also printed.

and $M_{2}$ for 5000 such Monte Carlo simulations, as well as the mean and standard deviation of the corresponding distribution. With this analysis, we find that $M_{1}=0.248 \pm 0.015$ and $M_{2}=0.096 \pm 0.006$, which is in excellent agreement with the results and associated uncertainties quoted in Table 6.

\section{SPECTROSCOPY}

In this section, we present the $J$ - and $K$-band spectra of GJ $164 \mathrm{AB}$. A careful characterization of the atmosphere and determination of abundances is necessary to understand the location of GJ $164 \mathrm{AB}$ in a mass-luminosity $(M / L)$ diagram, relative to an observational $M / L$ relation or to numerical simulations of M-dwarf atmospheres. The spectrum and absorption features of GJ $164 \mathrm{AB}$ were compared to the ones of other five M4-M5 dwarfs of known metallicity, aiming to determinate GJ $164 \mathrm{AB}$ overall abundance.

\subsection{Observations and Data Reduction}

Our spectroscopic sample consists of five M4-M5 dwarfs (in complement to GJ $164 \mathrm{AB}$ ) associated with solar-type stars, whose metallicity was measured by Valenti \& Fischer (2005). Assuming that both components formed from the same original molecular cloud, they should share the same metallicity. Their designation, spectral type, $(J-K)$ colors, as well as metallicities are listed in Table 7.

Near-infrared spectra of these objects were obtained with the recently commissioned TripleSpec spectrograph on the Palomar Hale Telescope (Wilson et al. 2004; Herter et al. 2008). TripleSpec at Palomar is a $\lambda / \Delta \lambda \approx 2500-2700$ cross-dispersed near-infrared spectrograph with a broad wavelength coverage across five simultaneous orders (1.0-2.4 $\mu \mathrm{m})$ in echelle format. Its entrance slit is $1 \times 30$ arcsec and a notable feature is that it has no moving parts.

The data were reduced with an IDL-based data reduction pipeline developed by P. Muirhead for TripleSpec at Palomar. The data were sky-subtracted using a sky-frame made by median combination of the five exposures on different positions of the object along the slit. To correct for telluric absorption features and flux-calibrate their spectra, an A0 V star was observed as close to the science object star airmass as possible. Each sky-subtracted exposure was then divided by a normalized flatfield, wavelength calibrated and fully extracted. The spectra are flux-calibrated and telluric corrected using the IDL-based code xtellcor_general described in the paper by Vacca et al. (2003).
Table 7

The Spectroscopic Sample

\begin{tabular}{lccc}
\hline \hline Name & Sp. Typ. & $\begin{array}{c}J-K \\
(\mathrm{mag})\end{array}$ & $\begin{array}{c}{[M / H]} \\
(\mathrm{dex})\end{array}$ \\
\hline GJ 324 B & M4 & 0.894 & $+0.25 \pm 0.03$ \\
GJ 544 B & M4 ${ }^{1}$ & 0.894 & $-0.15 \pm 0.03$ \\
GJ 611 B & M4 & 0.744 & $-0.49 \pm 0.03$ \\
GJ 166 C & M4.5 & 0.785 & $-0.08 \pm 0.03$ \\
NLTT 25869 & M5 & 0.872 & $+0.27 \pm 0.03$ \\
\hline
\end{tabular}

GJ 544 B is listed in SIMBAD as an M6.

\subsection{The Near-Infrared Spectrum of GJ 164}

The final $J$ - and $K$-band spectra of GJ $164 \mathrm{AB}$ and the five M4M5 dwarfs of known metallicity are shown in Figures 4 and 5, respectively. The equivalent width (EW) of prominent features were estimated using the IDL-based function measure_ew by N. Konidaris and J. Harker. Each spectrum was normalized and the EWs were calculated by computing the ratio of the area of a feature to a pseudocontinuum. This pseudocontinuum is a linear interpolation of the "clean" regions on either side of the feature. Uncertainties in the EW were obtained from the errors in the estimated pseudocontinuum and the errors in the measured intensities of each feature, as described in Sembach \& Savage (1992).

The EW of seven prominent features in $J$ band, shown in Figure 4, as well as Na I $(2.206 \mu \mathrm{m}$ and $2.209 \mu \mathrm{m})$, Ca I $(2.261 \mu \mathrm{m}, 2.263 \mu \mathrm{m}$, and $2.265 \mu \mathrm{m})$, and ${ }^{12} \mathrm{CO}(2,0)$ $(2.293 \mu \mathrm{m})$ in $K$ band are shown in Figure 6. Open circles depict the stars with $[M / H]<[M / H]_{\odot}(\mathrm{GJ} 544 \mathrm{~B}$, GJ $611 \mathrm{~B}$, and GJ $166 \mathrm{C})$ and filled circles depict the stars with $[M / H]>[M / H]_{\odot}$ (GJ 324 B and NLTT 25869). A star represents GJ 164 AB.

The EW of the features in the $K$ band of GJ $164 \mathrm{AB}$ (see Figure 6) seems to be compatible with the values of the stars with solar or above-solar metallicity in our sample. Its $K$-band spectrum differs appreciably from the one of GJ 611 (the lowest metallicity object in the sample), where all absorption features appear weaker compared with the rest of the sample, an expected manifestation of its low abundance nature. However, most of the features present in the spectra of these stars, especially in the $J$ band, are extremely temperature-dependent (Ali et al. 1995; Jones et al. 1996; except from the $\mathrm{Fe}$ I lines that do not exhibit any correlation with spectral type; Cushing et al. 2005), and prone to non-LTE effects, which make it difficult to assert unambiguously whether or not GJ $164 \mathrm{AB}$ is a metal-rich star. 


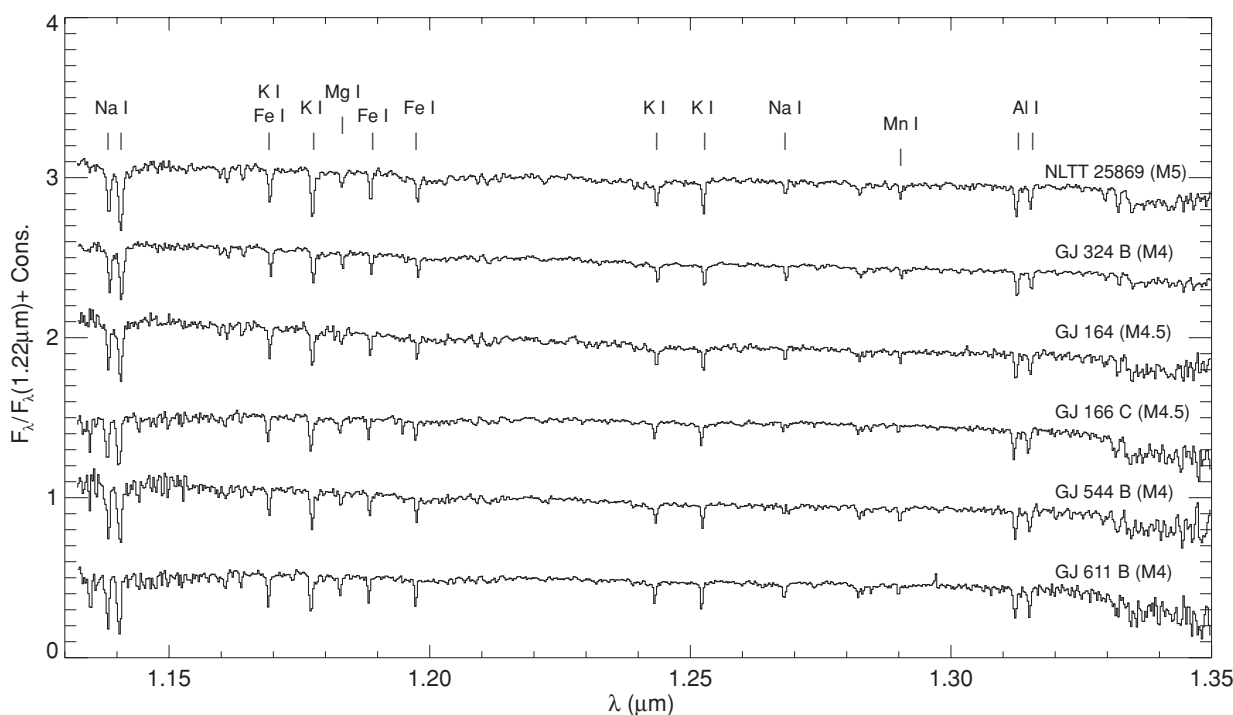

Figure 4. $J$-band spectra of the stars ordered from top to bottom by decreasing metallicity (listed in Table 7). The most prominent spectral features are highlighted.

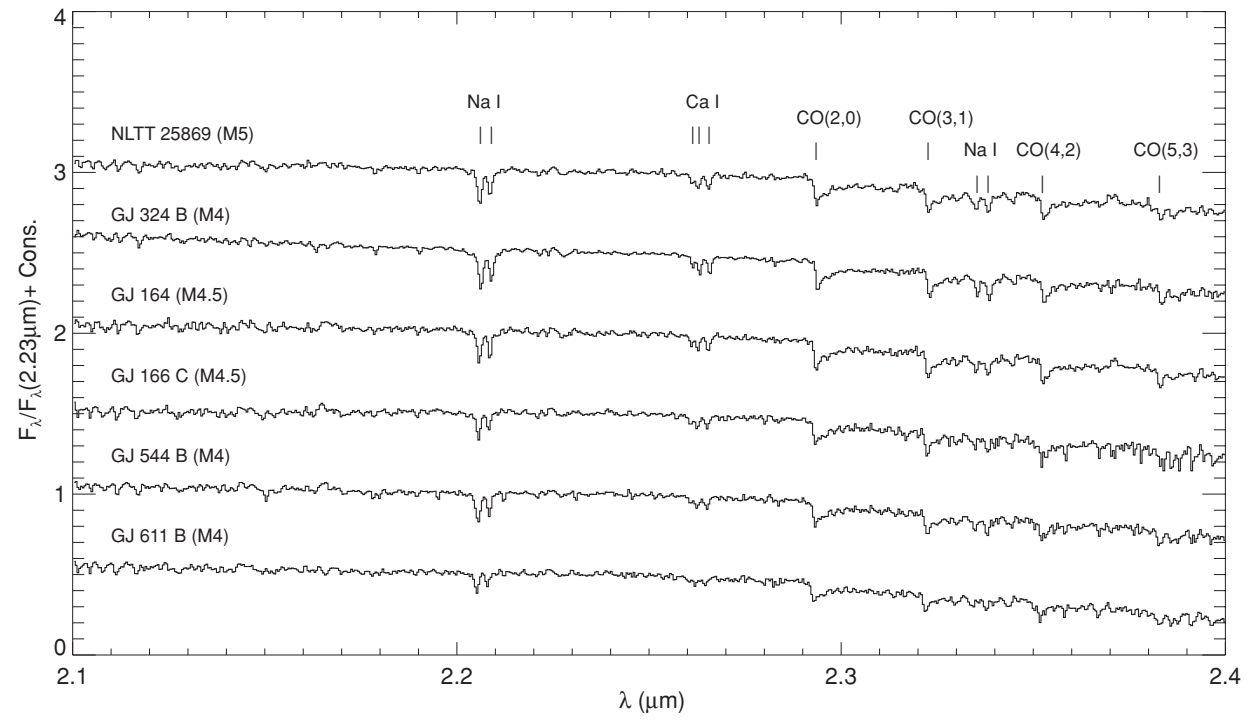

Figure 5. $K$-band spectra of the stars ordered from top to bottom by decreasing metallicity (listed in Table 7). The most prominent spectral features are highlighted.

\section{MASS-LUMINOSITY RELATIONS}

The individual apparent magnitudes calculated in Section 2.2, combined with the absolute parallax deduced from the dynamical analysis performed in Section 3.2, determine the following absolute $H$ and $K$ magnitudes: $M_{H}=7.719 \pm 0.047$ and $M_{K}=7.404 \pm 0.040$ for GJ $164 \mathrm{~A}, M_{H}=9.554 \pm 0.047$ and $M_{K}=9.121 \pm 0.052$ for GJ 164 B.

The $(H-K)$ colors, and to a lesser extent the infrared spectroscopy data presented in Section 4, indicate that GJ 164 is unlikely to be metal-poor. Figure 7 compares the location of GJ 164 A and B in a mass-luminosity diagram, relative to the low-mass solar metallicity models of Baraffe et al. (1998, hereafter referred to as BCAH98) for the $K$ band. For masses below $0.4 M_{\odot}$ (but above the substellar limit), models predict very little evolution between 0.5 and 5 Gyr. Figure 7 therefore only plots two isochrones, for 0.1 and $1 \mathrm{Gyr}$.

The aperture masking data places the primary less than $1 \sigma$ away from the $1 \mathrm{Gyr}$ isochrone, and $2 \sigma$ away from the $0.1 \mathrm{Gyr}$ isochrone. The hypothesis of an evolved age is supported by the kinematics of the system. Indeed, with the proper motion $(-324.5,-808.4)$ mas $\mathrm{yr}^{-1}$, the absolute parallax $\Pi=72 \pm 1.2$ mas derived in Section 3.2, as well as the radial velocity $V=-29.9 \mathrm{~km} \mathrm{~s}^{-1}$ measured by Pravdo et al. (2004), one can calculate the Galactic space velocity $(U, V, W)=$ $(-24,-20,-47) \mathrm{km} \mathrm{s}^{-1}$ after correction for standard solar motion. ${ }^{4}$ When compared to the properties of the nearest young moving groups (see Table 1 of López-Santiago et al. 2006), GJ 164 exhibits a velocity component perpendicular to the Galactic plane too large to be associated with any of these groups. From the kinematic properties of the M-Dwarf Survey of Reid et al. (2002), it is however impossible to conclude whether GJ 164 belongs to the thin or the thick disk. Figure 7 also plots the empirical $K$-band MLR established by Delfosse et al. (2000) for low-mass field stars, valid for $4.5<M_{K}<9.5$. Here again, the location of GJ 164 A seems in fairly good agreement with the MLR.

\footnotetext{
4 The sign convention is the one of the IDL astrolib gal_uvw procedure, with $U$ positive toward the Galactic anti-center, $V$ positive in the direction of Galactic rotation, and $W$ positive toward the North Galactic Pole.
} 

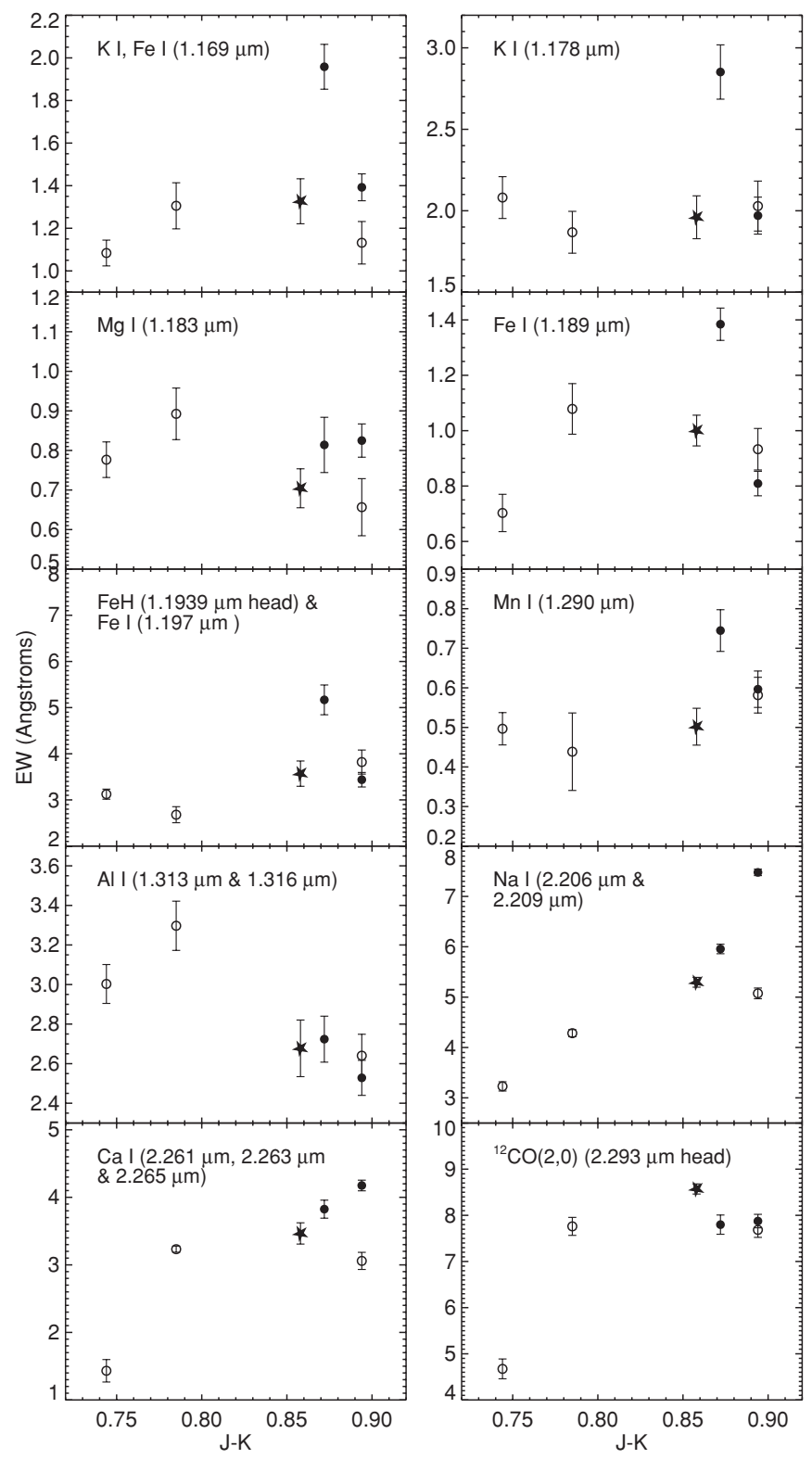

Figure 6. EWs of some of the most prominent lines in the sample spectra with $1 \sigma$ errors vs. $(J-K)$ colors. GJ 164 is represented by the five branch star, while metal-rich objects $([M / H]>0.0)$ are represented by filled circles and metal-poor objects $([M / H]<0.0)$ by open circles. The high value of GJ 164's ${ }^{12} \mathrm{CO}(2,0)(2.293 \mu \mathrm{m}) \mathrm{EW}$ may be attributed to its secondary. The 5:1 $\mathrm{K}$-band contrast ratio of the binary and the increasing strength of the $\mathrm{CO}$ bands with later types in the M-dwarf sequence, explain how GJ 164 B (unresolved with TripleSpec) may contribute to the strength of these spectral features.

The case of GJ $164 \mathrm{~B}$ is a little bit more puzzling. Indeed, with a mass $\lesssim 0.1 M_{\odot}$, GJ $164 \mathrm{~B}$ lies at the very end of the Delfosse et al. (2000) MLR, which makes comparisons with the MLR somewhat futile. The models however go well beyond. The reader will observe that as the mass decreases the luminosity they predict becomes a very steep function of the mass. Although GJ $164 \mathrm{~B}$ only lies $1 \sigma$ away from the $1 \mathrm{Gyr}$ isochrone, based on the dynamical mass and luminosity measurements reported in this work, the models seem to underestimate the luminosity of a $<0.1 M_{\odot}$ star by half a magnitude. A way to accommodate for this discrepancy would be for the GJ $164 \mathrm{~A}$ to be a tight binary, like for example the case of GJ 802 exposed in Ireland et al. (2008). Spectroscopic observations by Pravdo et al. (2004)

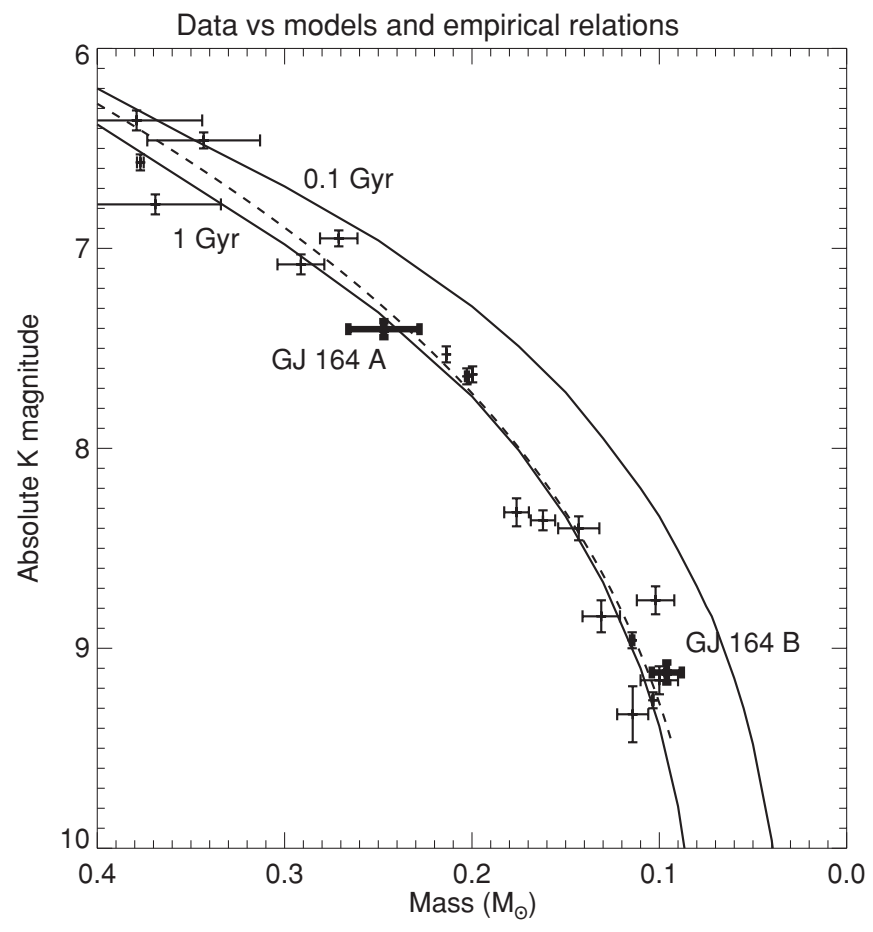

Figure 7. GJ $164 \mathrm{~A}$ and $\mathrm{B}$ in a mass-luminosity diagram (thick data points). The other data points were used by Delfosse et al. (2000) to establish an empirical $K$ band MLR for field stars (dashed line). The absolute $K$ magnitudes are compared to the BCAH98 isochrones for 0.1 and $1.0 \mathrm{Gyr}$ (two solid lines).

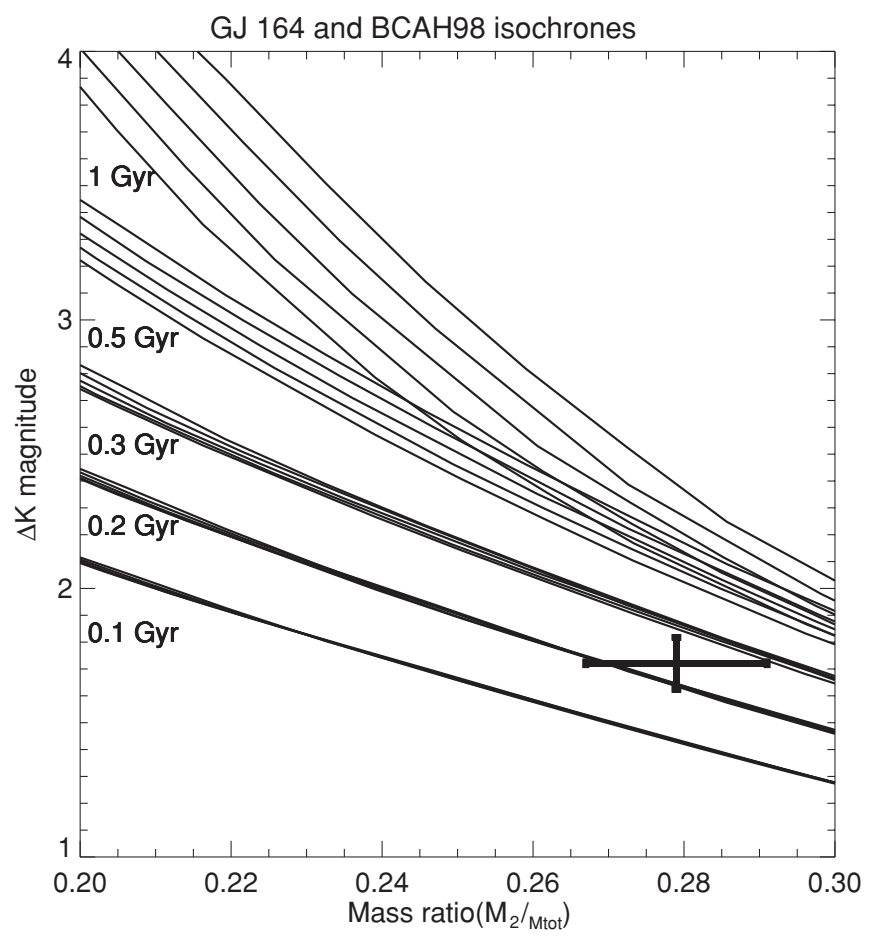

Figure 8. Mass ratio and $K$-band contrast ratio of GJ 164 (data point of coordinates $(0.25,1.8))$ are compared to BCAH98 isochrones for ages between 0.1 and 1 Gyr. Each age generates a family of models taking into account the uncertainty on the total mass of the binary.

however showed no evidence that GJ $164 \mathrm{~A}$ is a spectroscopic binary.

To provide a stronger case, Figure 8 plots more of the BCAH98 isochrones $(0.1,0.2,0.3,0.5$, and $1 \mathrm{Gyr})$, this time in the plane mass ratio $f$ (see Equation (4)), against the $K$-band magnitude difference. $\Delta K$ is a direct measurement made with 
the aperture masking interferometry data (see Table 2), while $f$ is a derived product of the joined fit, obtained in Section 3.2. Both observables are parallax-independent, which makes them more robust than the individual masses and luminosities.

Although taking into account all the uncertainties of our analysis by generating families of models within \pm 1 standard deviation of the total mass of the binary, the low significance discrepancy between the data and the models presented in Figure 7 now turns out to be $\sim 2 \sigma$ away from the $1 \mathrm{Gyr}$ isochrone: the models presented in Figure 8 clearly favor a very young age (250 Myr), which accommodates for the apparent excessive luminosity of GJ $164 \mathrm{~B}$, but is incompatible with the kinematics of the system. Note that the same conclusion holds when performing a similar analysis using $H$-band MLRs.

\section{CONCLUSION}

GJ 164 is an astrometric binary, whose orbit was resolved by aperture masking interferometry observations at Palomar and Keck. The consistency of the data is excellent, and exhibits a 12 mas precision in average for each measurement, made below what is usually accepted as the resolution limit of a telescope. These data, combined with earlier astrometric measurements, provide the following dynamical masses: $0.247 \pm 0.019 M_{\odot}$ for the primary and $0.096 \pm 0.008 M_{\odot}$ for the secondary.

Analysis of its infrared spectrum do not allow to unambiguously determine whether or not GJ $164 \mathrm{AB}$ is metal-rich. When attempting to use theoretical models for solar metallicity very low mass stars to derive an age from the mass and luminosity of both components, we find that the models do not adequately fit the data, requiring very young ages to accommodate for an overluminous secondary. There are only a handful number of dynamical masses for stars below $0.1 M_{\odot}$. The models are still waiting to be challenged in this critical region of the main sequence, right above the substellar limit.

The observations made here suggest that the models underestimate the luminosity of very low mass stars that have settled on the main sequence, but the precision of the dynamical data we present here, of the order of $10 \%$, however does not provide a strong constraint.

A precise measurement of the contrast ratio in the $R$ band, where the astrometry data was taken would already help to better constrain the mass ratio and ultimately improve the constraint on the individual masses, and make our statement about the models stronger. The very small separation (at most, 90 mas) makes this measurement difficult, even for Hubble Space Telescope. But because it lies so close from the substellar limit, GJ 164 B is a target that deserves most attention. In the meantime, a radial velocity curve would provide an independent measurement of the mass ratio. With an expected radial velocity amplitude of $2.8 \mathrm{~km} \mathrm{~s}^{-1}$, and a period of two years, observations of GJ 164 with TripleSpec and TEDI will settle this uncertainty.

We thank the staff and telescope operators of Palomar Observatory and Keck Observatory for their support. This work is partially funded by the National Science Foundation under grants AST-0504874 and AST-0705085. This publication makes use of the Simbad database, operated at CDS, Strasbourg, France and the data products from the 2MASS, which is a joint project of the University of Massachusetts and the Infrared Processing and Analysis Center/California Institute of Technology, funded by the National Aeronautics and Space Administration and the National Science Foundation. We extend special thanks to those of Hawaiian ancestry on whose sacred mountain we are privileged to be guests. Without their generous hospitality, the observations presented herein would not have been possible.

\section{REFERENCES}

Ali, B., Carr, J. S., Depoy, D. L., Frogel, J. A., \& Sellgren, K. 1995, AJ, 110, 2415

Baldwin, J. E., Haniff, C. A., Mackay, C. D., \& Warner, P. J. 1986, Nature, 320, 595

Baraffe, I., Chabrier, G., Allard, F., \& Hauschildt, P. H. 1998, A\&A, 337, 403

Baraffe, I., Chabrier, G., Barman, T. S., Allard, F., \& Hauschildt, P. H. 2003, A\&A, 402, 701

Burrows, A., Sudarsky, D., \& Hubeny, I. 2006, ApJ, 640, 1063

Cushing, M. C., Rayner, J. T., \& Vacca, W. D. 2005, ApJ, 623, 1115

Delfosse, X., Forveille, T., Ségransan, D., Beuzit, J.-L., Udry, S., Perrier, C., \& Mayor, M. 2000, A\&A, 364, 217

Hayward, T. L., Brandl, B., Pirger, B., Blacken, C., Gull, G. E., Schoenwald, J., \& Houck, J. R. 2001, PASP, 113, 105

Henry, T. J. 1998, in ASP Conf. Ser. 134, Brown Dwarfs and Extrasolar Planets, ed. R. Rebolo, E. L. Martin, \& M. R. Zapatero Osorio (San Francisco, CA: ASP) 28

Henry, T. J., Kirkpatrick, J. D., \& Simons, D. A. 1994, AJ, 108, 1437

Herter, T. L., et al. 2008, in Proc. SPIE 7014, Ground-based and Airborne Instrumentation for Astronomy II, ed. I. S. McLean \& M. M. Casali (Bellingham, WA: SPIE), 70140X

Ireland, M. J., Kraus, A., Martinache, F., Lloyd, J. P., \& Tuthill, P. G. 2008, ApJ, 678,463

Jones, H. R. A., Longmore, A. J., Allard, F., \& Hauschildt, P. H. 1996, MNRAS, 280,77

Kervella, P., et al. 2008, A\&A, 488, 667

Kirkpatrick, J. D., et al. 2000, AJ, 120, 447

Koornneef, J. 1983, A\&A, 128, 84

Kraus, A. L., Ireland, M. J., Martinache, F., \& Lloyd, J. P. 2008, ApJ, 679, 762

Lampton, M., Margon, B., \& Bowyer, S. 1976, ApJ, 208, 177

Liebert, J., \& Gizis, J. E. 2006, PASP, 118, 659

Lloyd, J. P., Martinache, F., Ireland, M. J., Monnier, J. D., Pravdo, S. H., Shaklan, S. B., \& Tuthill, P. G. 2006, ApJ, 650, L131

López-Santiago, J., Montes, D., Crespo-Chacón, I., \& Fernández-Figueroa, M. J. 2006, ApJ, 643, 1160

Martinache, F., Lloyd, J. P., Ireland, M. J., Yamada, R. S., \& Tuthill, P. G. 2007, ApJ, 661, 496

McCarthy, Jr, D. W., Henry, T. J., Fleming, T. A., Saffer, R. A., Liebert, J., \& Christou, J. C. 1988, ApJ, 333, 943

Monnier, J. D. 2000, in Principles of Long Baseline Stellar Interferometry, ed. P. R. Lawson (Pasadena, CA: NASA), 203

Nakajima, T., Kulkarni, S. R., Gorham, P. W., Ghez, A. M., Neugebauer, G., Oke, J. B., Prince, T. A., \& Readhead, A. C. S. 1989, AJ, 97, 1510

Nakajima, T., Oppenheimer, B. R., Kulkarni, S. R., Golimowski, D. A., Matthews, K., \& Durrance, S. T. 1995, Nature, 378, 463

Paczynski, B. 1986, ApJ, 304, 1

Pravdo, S. H., \& Shaklan, S. B. 1996, ApJ, 465, 264

Pravdo, S. H., Shaklan, S. B., Henry, T., \& Benedict, G. F. 2004, ApJ, 617, 1323

Pravdo, S. H., Shaklan, S. B., Wiktorowicz, S. J., Kulkarni, S., Lloyd, J. P., Martinache, F., Tuthill, P. G., \& Ireland, M. J. 2006, ApJ, 649, 389

Racine, R., Walker, G. A. H., Nadeau, D., Doyon, R., \& Marois, C. 1999, PASP, 111,587

Readhead, A. C. S., Nakajima, T. S., Pearson, T. J., Neugebauer, G., Oke, J. B., \& Sargent, W. L. W. 1988, AJ, 95, 1278

Reid, I. N., Gizis, J. E., \& Hawley, S. L. 2002, AJ, 124, 2721

Reid, I. N., Hawley, S. L., \& Gizis, J. E. 1995, AJ, 110, 1838

Sembach, K. R., \& Savage, B. D. 1992, ApJS, 83, 147

Tuthill, P. G., Monnier, J. D., Danchi, W. C., Wishnow, E. H., \& Haniff, C. A. 2000, PASP, 112, 555

Tuthill, P., et al. 2006, in Proc. SPIE 6272, Advances in Adaptive Optics II, ed. B. L. Ellerbroek \& D. Bonaccini Calia (Bellingham, WA: SPIE), 62723A

Vacca, W. D., Cushing, M. C., \& Rayner, J. T. 2003, PASP, 115, 389

Valenti, J. A., \& Fischer, D. A. 2005, ApJS, 159, 141

van de Kamp, P. 1971, ARA\&A, 9, 103

Weis, E. W. 1996, AJ, 112, 2300

Wilson, J. C., et al. 2004, in SPIE Conf. 5492, Ground-based Instrumentation for Astronomy, ed. A. F. M. Moorwood \& M. Iye (Bellingham, WA: SPIE), 1295 